\title{
Adaptive separation of random lines and background
}

\author{
Jürgen Beyerer \\ Fernando Puente León, MEMBER SPIE \\ Universität Karlsruhe \\ Institut für Meß- und Regelungstechnik \\ Postfach 6980 \\ D-76128 Karlsruhe \\ Germany \\ E-mail: beyerer@mrt.mach.uni-karlsruhe.de, \\ puente@mrt.mach.uni-karlsruhe.de
}

\begin{abstract}
An image processing method is presented that enables an efficient and robust separation of straight line-like structures from an isotropic background texture. The algorithm is based on a transform sequence mapping both image components onto two nearly disjoint and parameter invariant areas in the resulting transform domain. Applying a linear notch filter to the transformed image enables us to ascertain for each component of the discrete Fourier spectrum of the image, whether it contributes more to the line-like structures or more to the background. Based on this decision, the spectrum is split into two complementary and disjoint spectra, the inverse Fourier transforms of which are taken as estimates for both image components. In practical applications, the algorithm behaves robustly with respect to its parameters as well as to the assumptions made about the images. () 1998 Society of Photo-Optical Instrumentation Engineers. [S0091-3286(98)01410-X]
\end{abstract}

Subject terms: adaptive image enhancement; texture decomposition; line structure; optimal filtering; image processing; automated visual inspection.

Paper 980040 received Feb. 4, 1998; accepted for publication May 25, 1998.

\section{Introduction}

This paper describes an image processing method to separate randomly located straight line-like structures from an isotropic background texture. The lines are assumed to extend across the entire image, and the isotropy of the background means that the respective Fourier spectrum is approximately symmetric with respect to rotation, so that on spatial average, the background does not contain predominant directional structures. Examples where these assumptions are typically fulfilled are microscopic images of:

- technical and biological fiber structures

- toolmarks on machined surfaces

- particle traces in cloud or bubble chambers

- forensically relevant marks on firearm bullets and the like.

The method presented was developed especially for analyzing the surface texture of cylinders of combustion engines. Such surfaces are finished by honing-a machining process that generates several sets of parallel, straight, stochastically placed groove-shaped toolmarks. The entirety of the grooves constitutes a channel system that is responsible for retaining and distributing oil on the cylinder surface. Longevity, running-in behavior, oil consumption, and noxious emissions of the engine highly depend on the quality of the surface texture consisting of the honing grooves and a background texture, which is defined to contain all deviations from an ideal groove texture. Since different quality aspects are associated with the grooves and with the background, for an automatic assessment it is very advantageous to separate both components in a preprocessing step (see Fig. 8 in Section 4). For details, see Refs. 1, 2, and 3.

An obvious approach to accomplish the separation task would be, for example, to filter out the spectral components typical for line-like structures with heuristically defined linear filters. ${ }^{4}$ In the simplest case, the transfer functions of such filters are constructed using elementary formed regions as passbands and stop bands. This procedure yields good results, if the line-like structures are periodical and therefore well concentrated in the Fourier domain. However, if there are many lines at rather different angles, as on honed surfaces, the heuristic approach performs poorly. The reason for this is that the union of all the regions to be suppressed in the Fourier domain occupies a large area, and therefore many spectral components belonging to the background signal are also eliminated. In other words, the heuristic method tends to be too generous in defining the stop band for the line-like structures. Moreover, if the angles of the lines are unknown a priori, it is necessary to measure them to arrange an adequate transfer function based on a heuristically found filter for line structures of one known direction. $^{5}$

The method presented in this paper enables a robust, adaptive separation of the two image components by exploiting the spectral isotropy of the background and the anisotropy of line-like structures. For each image, an individual binary discrete Fourier transform (DFT) transfer function is generated. The passbands and stop bands are defined by deciding whether a spectral component contributes more to the background or more to the line-like structures. The decision is based on filtering the magnitude of the DFT by means of a transform sequence that maps both signal components onto two nearly disjoint regions. This transform sequence is inspired by a method used in Ref. 6 to suppress radial artifacts in positron emission tomography. The geometrical constellation of the resulting regions is essentially independent of the parameters of the line-like structures and the background. In particular, no measure- 


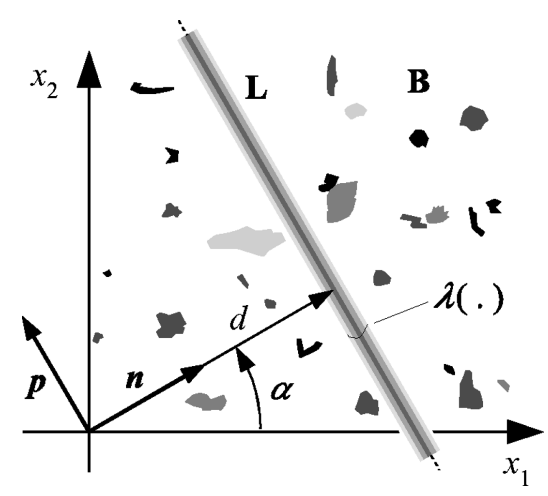

Fig. 1 Straight line embedded in an isotropic background.

ment of line angles is necessary, and the passbands and stop bands of the transfer function are defined point-bypoint without arbitrariness. The adaptive definition of a binary transfer function for each individual image can be regarded as an adaptive splitting of the DFT into two complementary spectra with disjoint support, where one is assigned to the background and the other to the line-like structures.

The theoretical foundations of our method are explained in the next section. In Section 3, we discuss in detail how the basic idea is turned into an image processing algorithm. The performance of the separation method is demonstrated in Section 4 with several examples.

\section{Signal Modeling}

To explain the basic idea, a simple signal model, which is reduced to essential assumptions, is used. The separation algorithm is designed for images $g(\mathbf{x})$ consisting of randomly placed straight line-like structures and an isotropic background $b(\mathbf{x})$. These two components may be combined additively or multiplicatively. Like in homomorphic filtering, ${ }^{7}$ the multiplicative case can be transformed into the additive case by taking the logarithm. To simplify the following considerations, but without restricting the generality, first only one single line embedded in a background is considered, and a continuous image signal of infinite extent is assumed. The signal model is:

$g(\mathbf{x})=\lambda\left(\mathbf{x}^{T} n-d\right)+b(\mathbf{x})$,

where $\lambda($.$) is the cross profile of the line, \alpha$ is the line angle, $n=(\cos \alpha, \sin \alpha)^{T}$ is the normal vector of the line, $p$ $=(-\sin \alpha, \cos \alpha)^{T}$ is a unit vector parallel to the line, $\mathbf{x}$ $=\left(x_{1}, x_{2}\right)^{T} \in \mathbb{R}^{2}$ is the location vector, and $d$ is the distance between the line and the origin of the $x_{1}, x_{2}$-domain. See Fig. 1, where $L$ and $B$ are used to symbolize the correspondence of signal components to the line and to the background $b(\mathbf{x})$, respectively.

The isotropy of $b(\mathbf{x})$ means that the magnitude of the corresponding Fourier transform is symmetric with respect to rotation, i.e.:

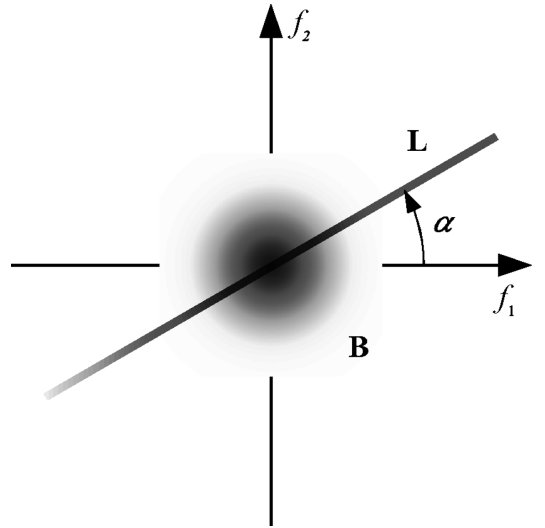

Fig. 2 Magnitude of $\mathscr{F}\{g(\mathbf{x})\}$.

$$
\begin{aligned}
B(\mathbf{f}) & =\mathscr{F}\{b(\mathbf{x})\} \\
& =\int_{-\infty}^{\infty} \int_{-\infty}^{\infty} b(\mathbf{x}) \exp \left(-\mathrm{j} 2 \pi \mathbf{f}^{T} \mathbf{x}\right) \mathrm{d} \mathbf{x} \\
& =B(\|\mathbf{f}\|),
\end{aligned}
$$

where $\|\mathbf{f}\|$ denotes the Euclidean norm of the spatial frequency vector $\mathbf{f}=\left(f_{1}, f_{2}\right)^{T} \in \mathbb{R}^{2}$ and $B($.$) the radial profile$ of $B(\mathbf{f})$.

In the following, a transformation sequence is given that maps the two components of $g(\mathbf{x})$ onto two almost disjoint areas, the shapes and locations of which are approximately independent of $d, \alpha, \lambda($.$) , and B($.$) . This independence can$ be interpreted as an invariance property with respect to the mentioned quantities. The transformation sequence forms the basis for the construction of the separation algorithm presented in this paper.

In the first step, the Fourier transform of $g(\mathbf{x})$ is $\operatorname{taken}^{8,9}$ :

$$
\begin{aligned}
\mathscr{F}\{g(\mathbf{x})\}= & G(\mathbf{f}) \\
= & \Lambda\left(\mathbf{f}^{T} n\right) \delta\left(\mathbf{f}^{T} p\right) \\
& \times \exp \left(-\mathrm{j} 2 \pi d \mathbf{f}^{T} n\right)+B(\|\mathbf{f}\|),
\end{aligned}
$$

with

$\Lambda(\eta)=\int_{-\infty}^{\infty} \lambda(\xi) \exp (-\mathrm{j} 2 \pi \eta \xi) \mathrm{d} \xi, \quad \eta \in \mathbb{R}$.

Since the $\delta$-function dominates $G(\mathbf{f})$ for $\mathbf{f}^{T} p=0$ and is zero elsewhere, the magnitude of Eq. (3) follows as:

$|G(\mathbf{f})|=\left|\Lambda\left(\mathbf{f}^{T} n\right)\right| \delta\left(\mathbf{f}^{T} p\right)+|B(\|\mathbf{f}\|)|$.

The removal of the phase, which is the second processing step, eliminates the dependence on the distance $d$. The result is illustrated in Fig. 2. The background $b(\mathbf{x})$ is transformed into a rotationally symmetrical component centered around the origin, and $\lambda\left(\mathbf{x}^{T} n-d\right)$ is concentrated on a straight line with normal vector $p$ through the origin of the $f_{1}, f_{2}$-domain. 


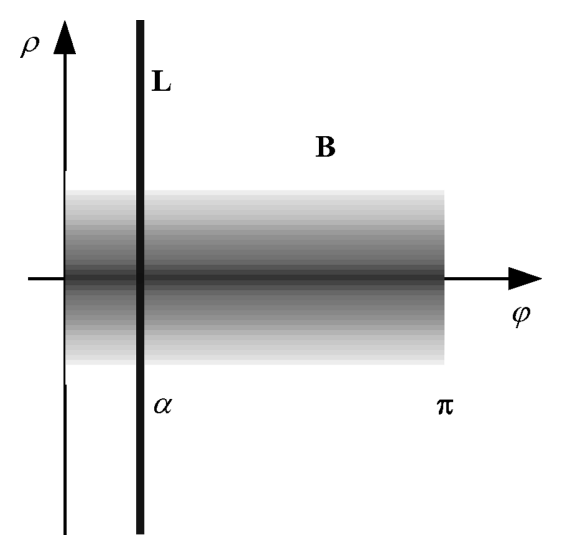

Fig. 3 Graph of $|G(\mathbf{f})|$ in a polar coordinate system.

In the next step, polar coordinates are introduced:

$$
\mathbf{f}=\rho(\cos \varphi, \sin \varphi)^{T}
$$

where $\varphi \in[0, \pi)$ is the angle of the spatial frequency vector and $\rho \in \mathbb{R}$ is the signed, radial frequency coordinate. Due to:

$$
\begin{aligned}
\delta\left(\mathbf{f}^{T} p\right) & =\delta[\rho(\sin \varphi \cos \alpha-\cos \varphi \sin \alpha)] \\
& =\delta\{\rho[\sin (\varphi-\alpha)]\}=\frac{\delta(\varphi-\alpha)}{|\rho|},
\end{aligned}
$$

$\rho \neq 0$

$\left.\mathbf{f}^{T} n\right|_{\mathbf{f}^{T} p=0}=\left.\rho(\cos \varphi \cos \alpha+\sin \varphi \sin \alpha)\right|_{\varphi=\alpha}=\rho$

and

$|\rho|=\|\mathbf{f}\|$

with respect to the $\rho, \varphi$-coordinate system, Eq. (4) becomes

$|G(\rho \cos \varphi, \rho \sin \varphi)|$

$$
=|\Lambda(\rho)| \frac{\delta(\varphi-\alpha)}{|\rho|}+|B(|\rho|)|, \quad \rho \neq 0 .
$$

Figure 3 shows that $\lambda\left(\mathbf{x}^{T} n-d\right)$ is mapped onto a $\delta$-line parallel to the $\rho$-axis and the background gathers round the $\varphi$-axis.

Now, Eq. (6) is multiplied by $|\rho|$ and the Fourier transform with respect to $(\rho, \varphi)$ is taken:

$$
\begin{aligned}
\mathscr{F}_{\rho, \varphi}[|\rho G(\rho \cos \varphi, \rho \sin \varphi)|] \\
=\mathscr{F}_{\rho}[|\Lambda(\rho)|] \mathscr{F}_{\varphi}\left[\sum_{k=-\infty}^{\infty} \delta(\varphi-\alpha-k \pi)\right] \\
\quad+\mathscr{F}_{\rho, \varphi}[|\rho B(|\rho|)|] \\
=\mathscr{F}_{\rho}[|\Lambda(\rho)|] \frac{1}{\pi} \sum_{k=-\infty}^{\infty} \delta\left(f_{\varphi}-\frac{k}{\pi}\right) \exp \left(-\mathrm{j} 2 \pi f_{\varphi} \alpha\right) \\
\quad+\mathscr{F}_{\rho}[|\rho B(|\rho|)|] \delta\left(f_{\varphi}\right) .
\end{aligned}
$$

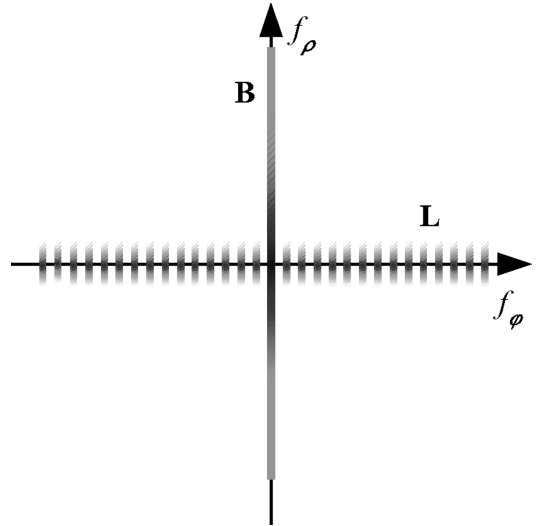

Fig. 4 Locations of background and straight line-like structures in the $f_{\rho}, f_{\varphi}$-domain.

As the integration in $\mathscr{F}_{\varphi}[]$ extends over $\varphi \in(-\infty, \infty)$, the periodicity due to the radial symmetry of $|G(\mathbf{f})|$ must be considered in Eq. (7). Figure 4 sketches the magnitude of the result in the $f_{\rho}, f_{\varphi}$-domain. The signal component $\lambda\left(\mathbf{x}^{T} n-d\right)$ mainly concentrates around the $f_{\varphi}$-axis, since for a thin line, i.e., for a short profile $\lambda(),. \mathscr{F}_{\rho}[|\Lambda(\rho)|]$ tends also to be of small extent with respect to $f_{\rho}$. The second term of Eq. (7) is equal to zero for $f_{\varphi} \neq 0$, and thus the background is mapped onto the $f_{\rho}$-axis.

The main features of the transform sequence shown here are that the intersection area of both signal components is small, and that the constellation of their supports in the $f_{\rho}, f_{\varphi}$-domain does not depend on the distance $d$ nor on the angle $\alpha$. Moreover, it is approximately independent of the line profile $\lambda($.) and the profile $B($.$) . It is easy to verify that$ these advantages remain preserved, even if several lines $\lambda_{i}\left(x_{1} \cos \alpha_{i}+x_{2} \sin \alpha_{i}-d_{i}\right)$ with different individual location parameters and profiles are present in $g(\mathbf{x})$. Further lines will be likewise mapped to vertical $\delta$-lines in the $\rho, \varphi$ domain and therefore will be concentrated around the $f_{\varphi}$-axis in the $f_{\rho}, f_{\varphi}$-domain; see also the examples in Section 4. So far, the $f_{\rho}, f_{\varphi}$-domain seems to be predestinated for the task of separating straight line-like structures from an isotropic background without utilizing any a priori knowledge concerning the line parameters. The invariant and almost disjoint manifestation of both image signal components should enable us to extract or suppress one of them within the $f_{\rho}, f_{\varphi}$-domain, and then to go back to the $x_{1}, x_{2}$-domain to obtain the separation result. Unfortunately, however, no inverse transform from the $f_{\rho}, f_{\varphi}$-domain back to the $x_{1}, x_{2}$-domain exists, because the phase information has been suppressed in Eq. (4) to obtain translation invariance.

To cope with this problem, the algorithm proposed in the next section suppresses signal components around the $f_{\varphi}$-axis and inverts the transform sequence only up to Eq. (4), resulting in a filtered version $|\widehat{B(f)}|$ of the magnitude $|G(\mathbf{f})|$. By comparing $|\widehat{B(\mathbf{f})}|$ and $|G(\mathbf{f})|$ for each spatial frequency $\mathbf{f}$, it can be decided whether the respective spectral component $G(\mathbf{f})$ contributes more to the background or more to the straight line-like structures of the image $g(\mathbf{x})$. 


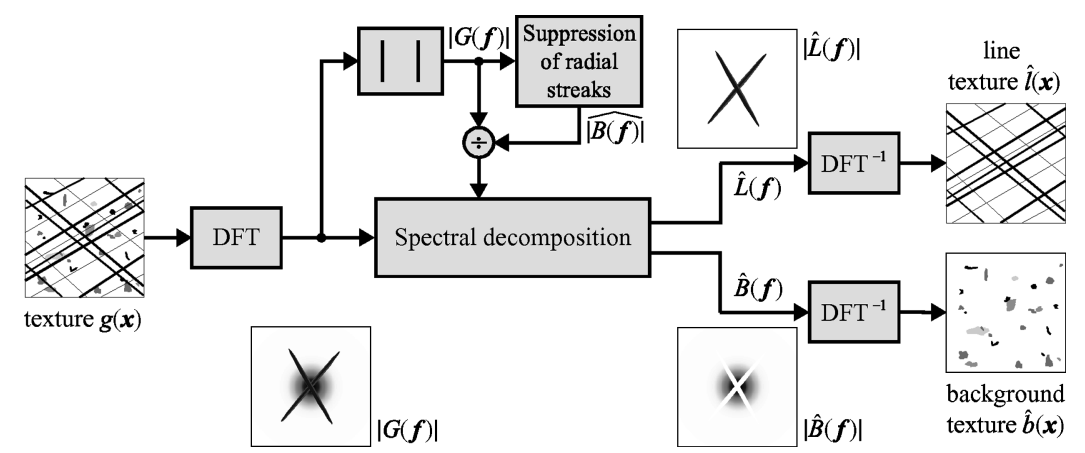

Fig. 5 Processing scheme for the separation of lines and background.

This is used to split the complex spectrum $G(\mathbf{f})$ into two disjoint spectra, the inverse Fourier transforms of which are estimates for the background texture and the straight line structures.

\section{Algorithm}

At this point, a comprehensive explanation of the signal processing algorithm used to separate lines and background is given. While in the former section image data were assumed to be continuous and of infinite extent, now the consequences of spatial sampling and restriction to a finite extent have to be taken into account. ${ }^{10}$ However, although small differences arise in how the data are treated, the basic considerations remain the same. Especially, the spatial restriction of the texture to a window corresponds to a convolution of the Fourier transform of the window with the Fourier transform of the texture. Thus, sharp structures in the frequency domain like $\delta$-functions are blurred.

We assume the discrete images to be quadratic with a size of $N \times N$ pixels. The texture images throughout this paper were digitized with $N \times N=256 \times 256$ pixels, and 8 bit gray levels. The following equations describe the relationship between continuous and discrete position and frequency vectors:

$\mathbf{x}=(x, y)^{T}=(i \Delta x, j \Delta y)^{T}$,

$\mathbf{f}=\left(f_{x}, f_{y}\right)^{T}=\left(\frac{k}{N \Delta x}, \frac{l}{N \Delta y}\right)^{T}$.

Figures 5 and 6 show the structure of the proposed signal processing method schematically. The 2-D DFT $G(\mathbf{f})$ of the discrete image $g(\mathbf{x})$ is computed by means of the fast Fourier transform (FFT) algorithm. ${ }^{10}$ Then, the magnitude $|G(\mathbf{f})|$ is filtered by the procedure depicted in Fig. 6, which provides for a suppression of radial streaks.

\subsection{Suppression of Radial Streaks}

First, a rectangular to polar coordinate transform is performed. The values of $|G(\rho \cos \varphi, \rho \sin \varphi)|$ on the discrete polar grid are calculated ${ }^{11}$ by linear interpolation of $|G(\mathbf{f})|$. This can be interpreted as sampling $|G(\mathbf{f})|$ on a polar grid.

It is essential to consider the choice of a sufficiently dense sampling grid to avoid aliasing effects. According to the sampling theorem of computer tomography, ${ }^{8}$ the interval $[0, \pi]$ must be sampled with at least $n$ angular steps $\Delta \varphi$ :

$n:=\frac{\pi}{\Delta \varphi} \geqslant \frac{\pi}{\sqrt{2}} W N$

where $W$ denotes the relative bandwidth of $g(\mathbf{x})$ with respect to the spatial sampling rate $(\Delta x)^{-1}=(\Delta y)^{-1}$. Since sampling with the Nyquist rate implies choosing $W=1, n$ $\geqslant 569$ is obtained. However, the texture images investi-

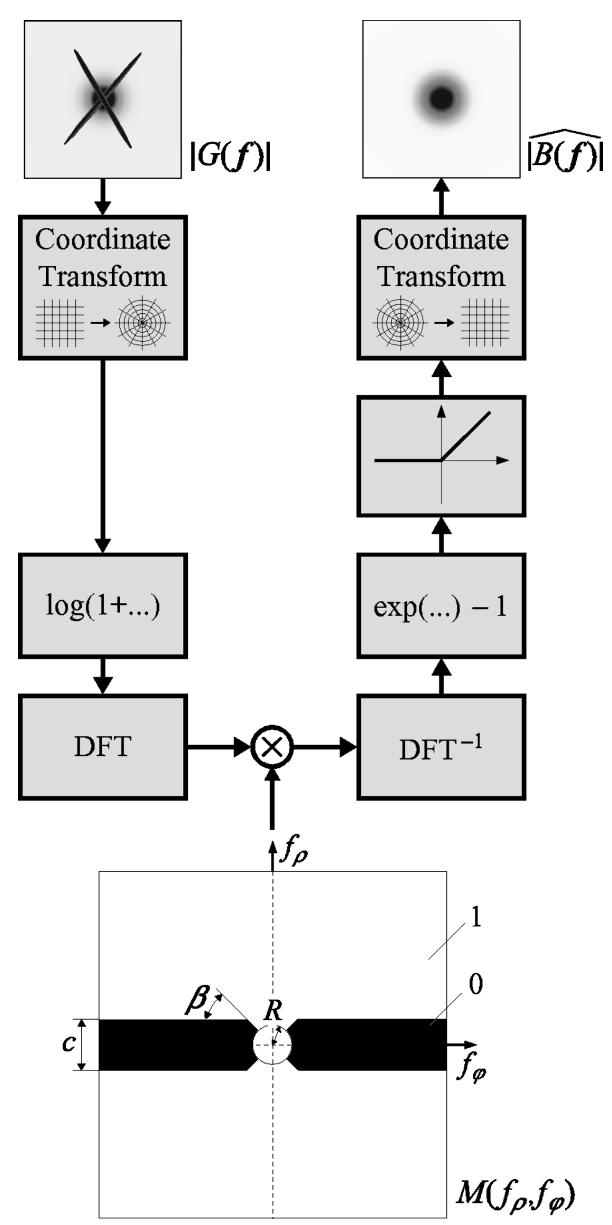

Fig. 6 Suppression of radial streaks. 
gated in this work are oversampled with $W \approx 0.8$ to 0.9 . To obtain an equivalent sampling for $\rho$, approximately $n$ radial steps are necessary. Therefore, for the number of angular as well as for the number of radial sampling steps, $n=512$ is chosen.

In the discrete case, the weight $|\rho|^{-1}$ of the $\delta$-function in Eq. (6) turns to a widening of the radial streaks of $|G(\mathbf{f})|$ for small values of $\rho$ after the polar coordinate transform, and therefore there is no need for compensating it. Since $g(\mathbf{x})$ is bandlimited, $|\Lambda(\rho)|$ usually decreases with increasing $\rho$ (see Fig. 3). If this decay could be compensated, the following Fourier transform $\mathscr{F}_{\rho, \varphi}[]$ would concentrate $L$ optimally onto the $f_{\varphi}$-axis. However, to keep the algorithm independent of the knowledge of $|\Lambda(\rho)|$, its dynamics are attenuated by applying a logarithm. Before this, the value 1 is added to avoid the singularity of the logarithm at zero (see Fig. 6).

The suppression of the streaks is performed by a linear filter in the $f_{\rho}, f_{\varphi}$-domain. The definition of a suitable filter mask is most crucial in the region around the $f_{\rho}, f_{\varphi}$-origin, because there the mappings of the lines and of the background are heavily superposed (see Fig. 4). Moreover, the origin carries the mean value of the signal to be filtered. This value is usually by far the highest one in the $f_{\rho}, f_{\varphi}$-domain. Due to the preprocessing by means of the log-function, the suppression of the component lying at the $f_{\rho}, f_{\varphi}$-origin causes an attenuation of the whole signal after inverting the logarithm. Therefore, the filter mask must let pass the component at the origin. The best results were achieved by using the notch filter mask $M\left(f_{\rho}, f_{\varphi}\right)$ shown in Fig. 6. To reduce edge effects in the $\rho, \varphi$-domain, the discontinuities of the notch filter are smoothed with a binomial low-pass filter. ${ }^{12}$ Choosing a simpler mask shape in the neighborhood of the $f_{\rho}, f_{\varphi}$-origin $(c=2 R, \beta=90 \mathrm{deg})$, as proposed in Ref. 6 , yielded unsatisfying results, because too many components belonging to the background were also suppressed. Since the subtraction of 1 after the inversion of the logarithm may lead to negative values, these are set to zero, if this occurs. After the transform back to rectangular coordinates, $|\widehat{B(\mathbf{f})}|$ is obtained, which is an estimate of the magnitude of the background spectrum.

\subsection{Spectral Decomposition}

Subsequently, the ratio $|G(\mathbf{f})| /|\widehat{B(\mathbf{f})}|$ is compared with a threshold $\gamma:=\sqrt{2}$ for each discrete frequency. If the ratio exceeds $\gamma$, the corresponding spectral component is assigned to the line pattern, otherwise to the background texture:

$$
\begin{aligned}
& \hat{L}(\mathbf{f})= \begin{cases}G(\mathbf{f}) & \text { if }|G(\mathbf{f})| /|\widehat{B(\mathbf{f})}|>\gamma \\
0 & \text { otherwise }\end{cases} \\
& \hat{B}(\mathbf{f})= \begin{cases}G(\mathbf{f}) & \text { if }|G(\mathbf{f})| /|\widehat{B(\mathbf{f})}| \leqslant \gamma \\
0 & \text { otherwise }\end{cases}
\end{aligned}
$$

The parameter choice for $\gamma$ is derived from the following fact: The Fourier transform $G(\mathbf{f})=L(\mathbf{f})+B(\mathbf{f})$ at the discrete frequency $\mathbf{f}$ contributes more to the line texture, if the spectral power of $l(\mathbf{x})$ predominates, i.e., if the condition:

$\frac{|L(\mathbf{f})|^{2}}{|B(\mathbf{f})|^{2}}>1$

holds. With the arguments leading to Eq. (4)

$|G|^{2}=|L+B|^{2}=|L|^{2}+L^{*} B+L B^{*}+|B|^{2} \approx|L|^{2}+|B|^{2}$

follows, and with $|\widehat{B(\mathbf{f})}|$ as an estimate of $|B(\mathbf{f})|$ we obtain:

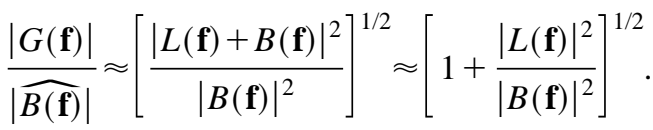

Therefore, Eq. (13) is approximately equivalent to the postulation:

$\frac{|G(\mathbf{f})|}{|\widehat{B(\mathbf{f})}|}>\sqrt{2}$

These considerations enable to define the threshold $\gamma$ theoretically, avoiding the need of making an empirical choice. Their validity was verified by means of optimization based on synthetic textures. ${ }^{2}$ Moreover, in practice this choice for $\gamma$ has proven to be very robust for a wide range of real textures (see Section 4).

As a result, the procedure delivers estimates $\hat{L}(\mathbf{f})$ and $\hat{B}(\mathbf{f})$ of the Fourier spectra of the line texture-denoted by $l(\mathbf{x})$ - and of the background $b(\mathbf{x})$, respectively. Finally, the estimates $\hat{l}(\mathbf{x})$ and $\hat{b}(\mathbf{x})$ of the line texture and of the background are obtained by inverse DFT of the spectra $\hat{L}(\mathbf{f})$ and $\hat{B}(\mathbf{f})$.

Since $G(\mathbf{f})=\hat{L}(\mathbf{f})+\hat{B}(\mathbf{f})$, and therefore also $g(\mathbf{x})$ $=\hat{l}(\mathbf{x})+\hat{b}(\mathbf{x})$ holds, the method implicitly assumes an additive composition $g(\mathbf{x})=l(\mathbf{x})+b(\mathbf{x})$ of the line texture and the background. Thus, it is sufficient to compute $\hat{L}(\mathbf{f})$ and its inverse DFT $\hat{l}(\mathbf{x})$, and to estimate the background by using:

$\hat{b}(\mathbf{x})=g(\mathbf{x})-\hat{l}(\mathbf{x})$.

If it is reasonable to assume that $l(\mathbf{x})$ and $b(\mathbf{x})$ are combined multiplicatively, the algorithm can be applied to $\log [g(\mathbf{x})]$.

\subsection{Remark}

At this point, the question may arise if it is not possible to apply the suppression of radial streaks directly to the complex spectrum instead of applying it to its magnitude. The answer is that the real and imaginary parts of the Fourier transform of line textures are heavily modulated. The radial streaks corresponding to the straight line-like structures look less compact-i.e., more interrupted-than the streaks in the magnitude of the spectrum. Thus, applying the trans- 


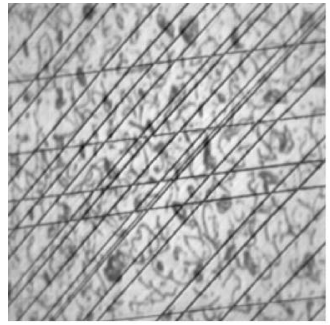

(a)

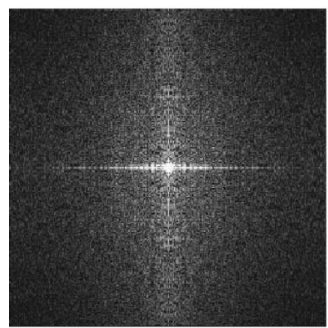

(d)

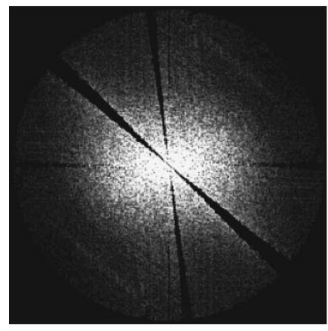

(g)

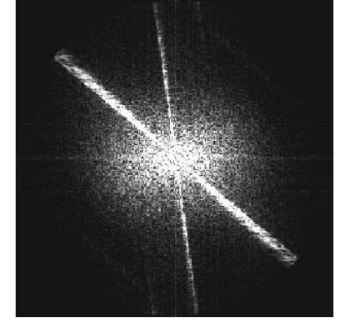

(b)

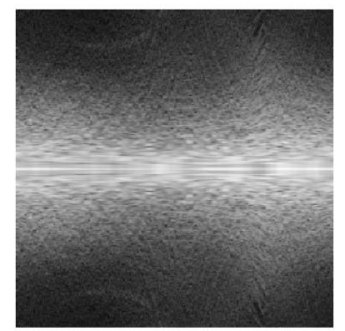

(e)

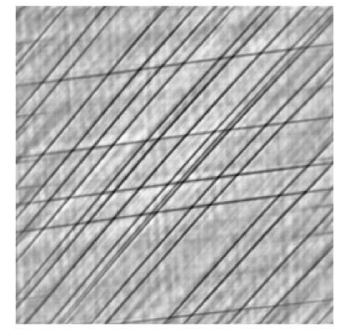

(h)

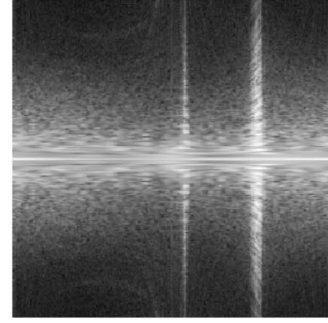

(c)

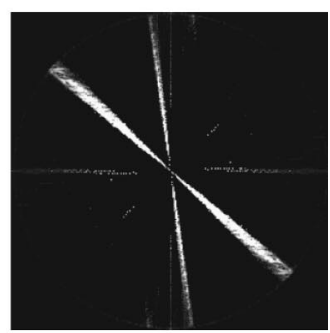

(f)

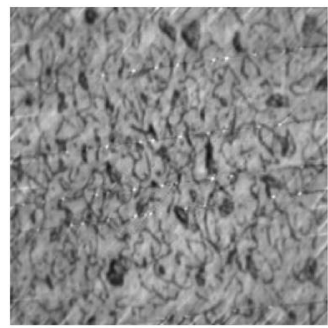

(i)

Fig. 7 Test picture: (a) original image; (b) $|G(\mathbf{f})|$; (c) $\log (|G(\mathbf{f})|+1)$ in polar coordinates, reflected on the $\varphi$-axis (horizontal); (d) DFT of (c); (e) DFT ${ }^{-1}$ of $(\mathrm{d}) \times M\left(f_{\rho}, f_{\varphi}\right)$; (f) magnitude $\hat{L}(\mathbf{f})$ of the line spectrum; (g) magnitude $\hat{B}(\mathbf{f})$ of the background spectrum; (h) line texture $\hat{l}(\mathbf{x})$; (i) background texture $\hat{b}(\mathbf{x})$.

form sequence to the Fourier transform of the texture would lead to a much lower concentration of the line information in the $f_{\rho}, f_{\varphi}$-domain.

\section{Experimental Results}

To obtain a proper choice for the parameter values of the notch filter mask $M\left(f_{\rho}, f_{\varphi}\right)$, a minimization of the error arising from the separation of lines and background was performed. For that purpose, a synthetic test picture was composed by adding a hand-drawn line texture, and a background texture [see Fig. 7(a)]. The minimization procedure delivered the following optimal mask parameters: $R=2, c$ $=8$ (both in pixels), and $\beta \approx 20 \mathrm{deg}{ }^{2}{ }^{2}$ Of course, these parameters depend on the texture under view, but the minimum has proven to be fairly flat, and it shows very weak dependence on the parameters for their range of interest. Although $R$ is chosen as small as possible, the component at the $f_{\rho}, f_{\varphi}$-origin should remain unchanged. Since the mask is smoothed with a $3 \times 3$ binomial low-pass, ${ }^{12} R=2$ is chosen.

Figure 7 shows the processing of the test picture with all intermediate results. The parameters used are: $R=2, c$ $=8$, and $\beta=20 \mathrm{deg}$. The comparison of Fig. 7(a) with Figs. 7(h) and 7(i) demonstrates the high performance of the proposed algorithm. In addition, Figs. 7(b) to 7(g) enable a comparison of the analogy between the theoretical considerations discussed in Section 2, and the processing of a discrete signal.

To show the relevance of the separation method to practical inspection tasks, results obtained with real textures are presented. Note that the proposed algorithm behaves very robustly for a broad range of real textures, even if the assumptions made in Section 2 are only partly fulfilled: in all cases, the parameters of the notch filter mask were chosen exactly as in Fig. 7.

Figure 8 shows the result of separating grooves and background for a honing texture. With Fig. 8(b) it can be seen that in the background image objects and defects of honing surfaces are enhanced very efficiently. Especially, defects at groove edges, and groove interrupts, which belong to the background, are extracted accurately. In contrast to this, since the grooves contained in the groove image Fig. 8(c) are smooth along their course, this image can be used for the exact estimation of the parameters of individual grooves. Based on both separation results, a method was developed allowing a robust detection of defective grooves. ${ }^{1}$

With the bullet area depicted in Fig. 9(a), a forensic application of the algorithm is demonstrated. Due to the 


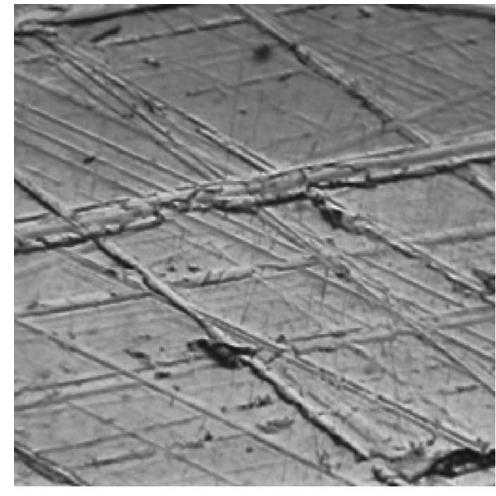

(a)

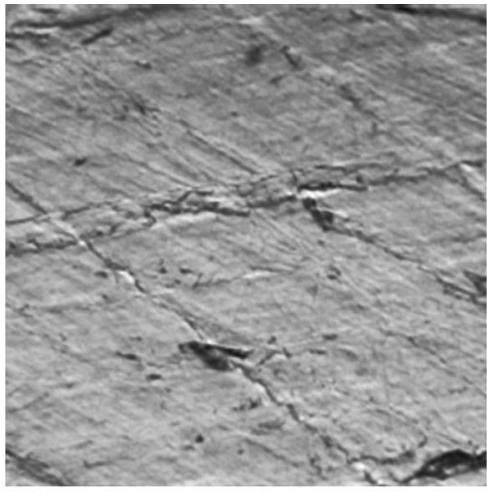

(b)

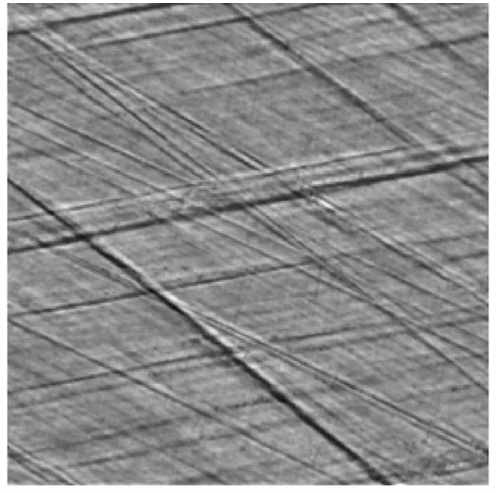

(c)

Fig. 8 Honed surface: (a) original image, (b) background image, and (c) groove image.

kinematics of the firing process, groove-shaped traces are generated by a firearm on the circumferential surface of the bullet. These grooves can be considered as a "fingerprint", of the gun and, consequently, they can be used for its identification. To match the images of two different bullets, the groove information is used, whereas the background information can be regarded as a disturbing component. The separation results show a clear discrimination between the disturbing component Fig. 9(b) and the signal of interest Fig. 9(c), so that by using this algorithm as a preprocessing tool, a higher robustness in a later automatic comparison step can be expected.

The next example is concerned with detection of defects in fabrics. Figure 10(a) shows the original image of a fabric containing several warp float defects. By means of the separation algorithm, the image can be decomposed into two different partial textures, namely the background including the defects [Fig. 10(b)] and the ideal texture [Fig. $10(\mathrm{c})]$. Since the background is clearly less complex than the original texture, the detection of defects based on this image is comparatively easier. Note also that the separation method performs very robustly, although the line-like structures differ considerably from the assumptions made in Section 2.
In many biological applications, microscopic images of translucent objects acquired with back-lighting are to be examined. An example showing a detail of a fowl feather is depicted in Fig. 11(a). Since in such cases a multiplicative combination of $l(\mathbf{x})$ and $b(\mathbf{x})$ must be assumed, the separation algorithm is applied to $\log [g(\mathbf{x})]$, and subsequently an inversion of the logarithm is performed. Although, as before, some of the assumptions made in Section 2 are not fulfilled, the method behaves quite robustly. Especially, note that the subtle structures along the feather fibers have been assigned to the background texture Fig. 11(b), whereas the lines in the complementary image Fig. 11(c) look much more uniform.

\section{Discussion}

From a more abstract point of view, the method presented can be interpreted as an optimal filter with respect to a specific constraint. Let $T(\mathbf{f})$ be the transfer function of a linear filter used to extract $l(\mathbf{x})$ in an optimal way:

$$
\hat{l}(\mathbf{x})=\operatorname{DFT}^{-1}[\hat{L}(\mathbf{f})]=\operatorname{DFT}^{-1}[T(\mathbf{f}) G(\mathbf{f})],
$$

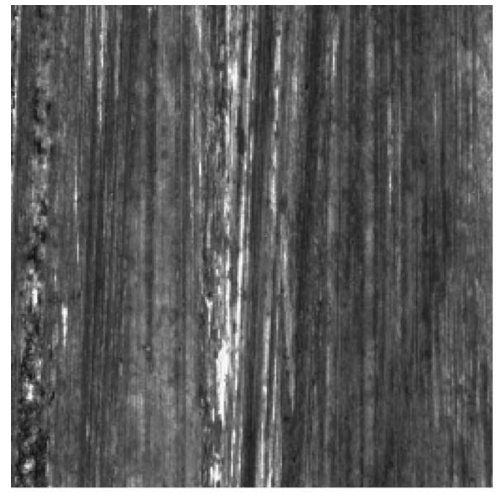

(a)

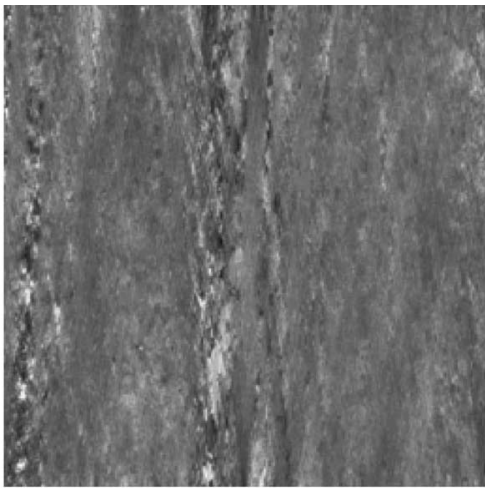

(b)

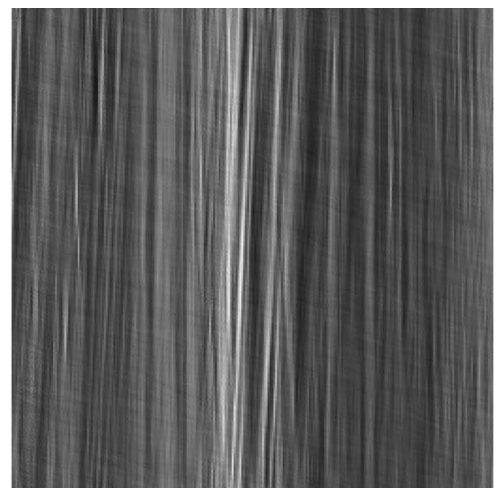

(c)

Fig. 9 Firearm bullet surface: (a) original image, (b) background image, and (c) groove image. 


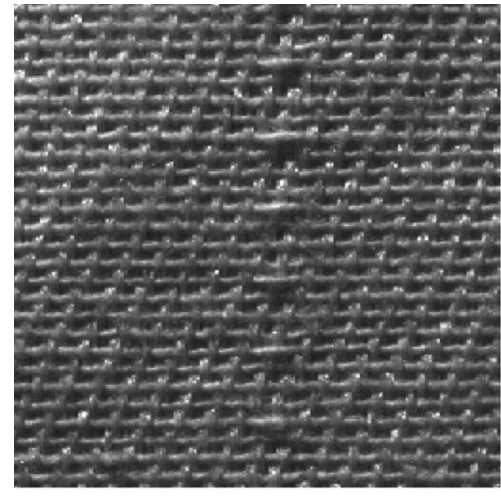

(a)

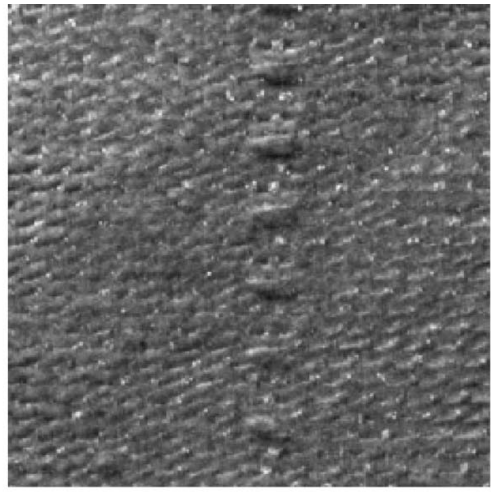

(b)

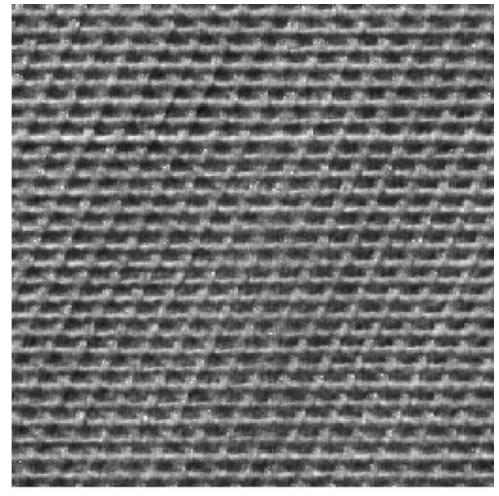

(c)

Fig. 10 Fabric: (a) original image, (b) background image, and (c) line image.

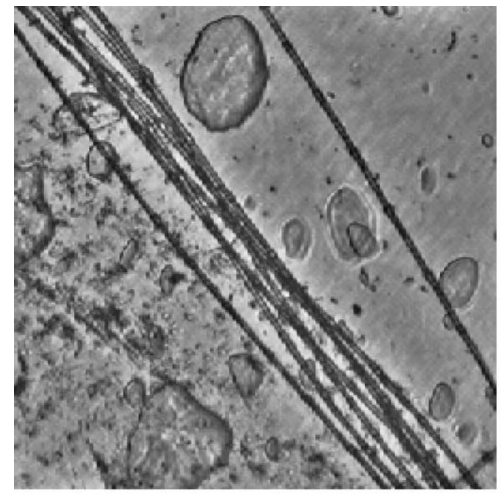

(a)

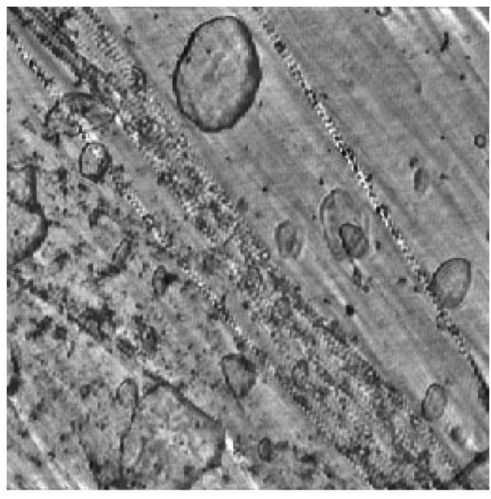

(b)

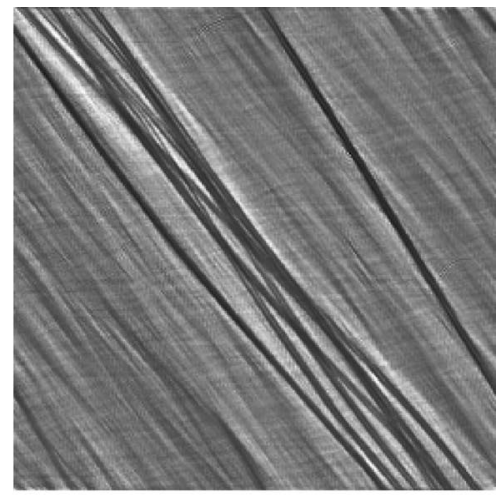

(c)

Fig. 11 Fowl feather: (a) original image, (b) background image, and (c) line image.

if $g(\mathbf{x})=l(\mathbf{x})+b(\mathbf{x})$ is assumed. As optimization criterion, we choose the mean squared error $e$ :

$e:=\sum_{\mathbf{x}}[l(\mathbf{x})-\hat{l}(\mathbf{x})]^{2}=\sum_{\mathbf{f}}|L(\mathbf{f})-\hat{L}(\mathbf{f})|^{2}$,

which can be calculated either in the spatial domain or, by virtue of Parseval's theorem, ${ }^{10}$ in the frequency domain. Using the second of these alternatives, we obtain:

$e=\sum_{\mathbf{f}}|L-T G|^{2}=\sum_{\mathbf{f}}|(1-T) L-T B|^{2}$.

Restricting $T(\mathbf{f})$ to a binary transfer function which can take only the values 0 and 1 , the error becomes

$$
\begin{aligned}
e & =\sum_{\mathrm{f}}[(1-T)^{2}|L|^{2}-\underbrace{1-T) T}_{=0}\left(L^{*} B+L B^{*}\right)+T^{2}|B|^{2}] \\
& =\sum_{\mathrm{f}}\left[(1-T)^{2}|L|^{2}+T^{2}|B|^{2}\right] .
\end{aligned}
$$

Obviously, the minimum of Eq. (21) is achieved for:
$T_{\{0,1\}}(\mathbf{f})=\left\{\begin{array}{ll}1 & \text { for }|L(\mathbf{f})|^{2}>|B(\mathbf{f})|^{2} \\ 0 & \text { otherwise }\end{array}\right.$.

Thus, the optimal filter is equivalent to the spectral decomposition in Subsection 3.2.

One might object that the constraint $T(\mathbf{f}) \in\{0,1\} \forall \mathbf{f}$ is too restrictive, and that one could get better results with weaker or no constraints. For example, if we assume $T(\mathbf{f}) \in \mathbb{R} \forall \mathbf{f}$, with the approximation of Eq. (14) and by using calculus, minimization of $e$ yields the optimal transfer function

$T_{\mathbb{R}}(\mathbf{f})=\frac{|L(\mathbf{f})|^{2}}{|L(\mathbf{f})|^{2}+|B(\mathbf{f})|^{2}} \approx \frac{|L(\mathbf{f})|^{2}}{|G(\mathbf{f})|^{2}}$.

In practice, however, the first approach performs much better with respect to the visual quality of the separation. One reason might be that the human visual system applies quality criteria different from the mean squared error. A further explanation can be found in the dominant role the phase information plays in image data. ${ }^{13}$ Because in the second approach the filter does not totally eliminate the phase information of spectral components which contribute more to 
the background texture, in $\operatorname{DFT}^{-1}\left[T_{\mathrm{R}}(\mathbf{f}) G(\mathbf{f})\right]$ details of the background are much stronger visible than in $\operatorname{DFT}^{-1}\left[T_{\{0,1\}}(\mathbf{f}) G(\mathbf{f})\right]$.

To adapt both filter types to individual images, the suppression of radial streaks is needed to reliably estimate $|B(\mathbf{f})|^{2}$ and with Eq. (14) also $|L(\mathbf{f})|^{2} \approx|G(\mathbf{f})|^{2}-|\widehat{B(\mathbf{f})}|^{2}$.

\section{Summary}

In this paper, a signal processing algorithm enabling the separation of straight line-like structures from an isotropic background texture has been presented. The discrimination between lines and background is enabled by a transform sequence that maps both image components onto two nearly disjoint areas in the resulting transform domain.

After having introduced a suitable signal model, the underlying theoretical foundations of the method have been discussed. Subsequently, a comprehensive description of the algorithmic implementation has been given. Despite the differences arising due to the processing of discrete images, the algorithm leads to fully satisfactory results.

To verify the usefulness of the proposed method, it has been applied to several practically relevant real textures. In all cases, the algorithm has proven to be a powerful preprocessing tool within the analysis of textures containing linelike structures. It behaves very robustly with respect to its parameters as well as to the assumptions made about the images.

Finally, at a more abstract level, we have also shown that the proposed method can be interpreted as an optimal filter with respect to a specific constraint.

\section{References}

1. J. Beyerer and F. Puente León, "Detection of defects in groove textures of honed surfaces," Int. J. Mach. Tools Manufact. 37(3), 371389 (1997).

2. J. Beyerer, "Suppression of stochastically placed, straight toolmarks to enhance objects and defects," Tech. Messen 59(10), 389-397 (1992).

3. J. Beyerer, "Model-based analysis of groove textures with applications to automated inspection of machined surfaces,' Measurement 15, 189-199 (1995).
4. C. W. Carrol, N. W. H. Sufi and R. C. Chang, "Suppression of tool marks to enhance detection of surface defects," in Surface Characterization and Testing II, M. Young and J. E. Greivenkamp, Ed., Proc. SPIE 1164, 212-221 (1989).

5. J. Beyerer, Analyse von Riefentexturen, Reihe 8, No. 390, VDIVerlag, Düsseldorf (1994).

6. M. A. Abidi and P. B. Davis, "Radial noise filtering in positron emission tomography," Opt. Eng. 29(5), 567-574 (1990).

7. R. C. Gonzalez and R. E. Woods, Digital Image Processing, AddisonWesley, Reading, MA (1993).

8. R. Bamler, Mehrdimensionale Lineare Systeme, Springer-Verlag, Berlin (1989).

9. R. N. Bracewell, The Fourier Transform and Its Applications, McGraw-Hill, Tokyo (1978).

10. E. O. Brigham, The Fast Fourier Transform and Its Applications, Prentice Hall, Englewood Cliffs, NJ (1988).

11. W. K. Pratt, Digital Image Processing, John Wiley \& Sons, New York (1991).

12. B. Jähne, Digital Image Processing-Concepts, Algorithms, and Scientific Applications, Springer-Verlag, Berlin (1997).

13. A. V. Oppenheim, "The importance of phase in signals," Proc. IEEE 69(5), 529-541 (1981).

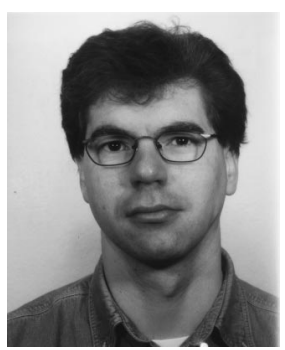

Jürgen Beyerer received an MS degree in electrical engineering in 1989 and a $\mathrm{PhD}$ degree in automated visual inspection in 1994, both from the University of Karlsruhe, Germany. He is currently a postdoctoral research associate at the Institut für Meß und Regelungstechnik of the University of Karlsruhe. His research interests are measurement theory, information theory, statistical signal processing, image processing, and automated visual inspection.

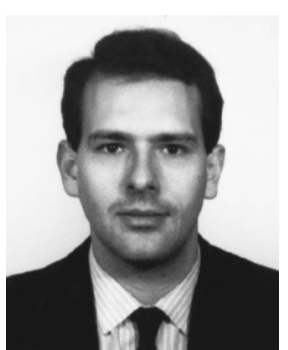

Fernando Puente León received an MS degree in electrical engineering in 1994 from the University of Karlsruhe, Germany. Since May 1994, he has been a research assistant with the Institut für Meß und Regelungstechnik of the University of Karlsruhe. His research interests include signal processing, automated visual inspection, and pattern recognition. He is currently working on the development of image processing methods for the identification of firearms. 